\title{
Skiciranje grafova nekih složenih trigonometrijskih funkcija
}

Mandi Orlić Bachler, Luka Marohnić, Martina Dokić

\section{Sažetak}

U radu se opisuje kako se na elementaran način (bez primjene diferencijalnoga računa, te praktički koristeći samo papir i olovku) mogu na nekom segmentu $[a, b] \subset \mathbb{R}$ skicirati grafovi nekih složenih trigonometrijskih funkcija oblika

$$
\begin{aligned}
& f(x)=A \sin (g(x)), \\
& f(x)=A \cos (g(x)), \\
& f(x)=g(x) \cdot \sin (\omega x+\varphi), \\
& f(x)=g(x) \cdot \cos (\omega x+\varphi),
\end{aligned}
$$

gdje su $A, \omega>0$ i $\varphi \in[-\pi, \pi]$ realne konstante, a $g: \mathbb{R} \rightarrow\langle 0,+\infty\rangle$ kvadratna ili eksponencijalna funkcija.

Ključni pojmovi: trigonometrijske funkcije, grafovi, skiciranje

\section{Uvod}

U sklopu matematičkih i strukovnih kolegija na visokoškolskim ustanovama, studenti se često susreću sa zadacima u kojima je potrebno skicirati graf funkcije koji služi kao zorna pomoć u rješavanju zadanog problema. Najčešće, skiciranje grafa temelji se na ispitivanju toka funkcije, a taj posao zna vrlo često biti dugačak i složen, pogotovo kada se

Ovaj rad je podržan od strane Hrvatske zaklade za znanost (HRZZ) u okviru projekta UIP-05-2017-9219. 
radi o složenim funkcijama poput ovih koje ćemo promatrati u radu, a koje su oblika:

$$
\begin{array}{rll}
f(x)=A \sin (g(x)) & \text { odnosno } & f(x)=A \cos (g(x)), \text { te } \\
f(x)=g(x) \cdot \sin (\omega x+\varphi) & \text { odnosno } & f(x)=g(x) \cdot \cos (\omega x+\varphi),
\end{array}
$$

gdje su: $A, \omega>0$ i $\varphi \in[-\pi, \pi]$ realne konstante. Zbog jednostavnosti za funkciju $g$ promatrat ćemo samo one oblike funkcija čije grafove nije teško nacrtati (primjerice $g(x)=e^{-x}$ ili $g(x)=1+x^{2}$ ). Prema tome, neka je $g: \mathbb{R} \rightarrow\langle 0,+\infty\rangle$ funkcija oblika

$$
g(x)=\alpha x^{2}+\beta x+\gamma, \quad \text { gdje je } \quad \alpha>0 \quad \text { i } \quad \beta^{2}-4 \alpha \gamma<0
$$

ili oblika

$$
g(x)=e^{\alpha x+\beta},
$$

gdje su $\alpha, \beta$ i $\gamma$ realne konstante. Stoga, kako bismo skiciranje sveli na brz i rutinski posao, opisat ćemo metode koje se sastoje u tome da se, kao pomoćno sredstvo za skiciranje grafa funkcije $f$, najprije konstruira graf funkcije $g$ (što je značajno lakše i jednostavnije za učiniti u odnosu na konstruiranje grafa funkcije $f$ ).

Postupci konstruiranja grafova funkcija $g$ u radu neće biti ni objašnjeni, ni dokazani, ni popraćeni odgovarajućim crtežima. Te funkcije se obrađuju u sastavu nastavnih programa matematike u gimnazijama, ali i u sastavu nastavnih programa matematičkih predmeta na većini visokoobrazovnih ustanova u Republici Hrvatskoj. O svojstvima funkcija $g$, kao i postupak konstrukcije njihovih grafova, može se pronaći u literaturi [1, 2, 3].

Rad je podijeljen u dva dijela. U prvom dijelu rada opisan je postupak skiciranja grafova funkcija oblika (1), a u drugom dijelu funkcija oblika (2).

\section{Graf funkcije oblika $f(x)=A \sin (g(x))$}

Postupak skiciranja grafa funkcije $f: \mathbb{R} \rightarrow[-A, A]$ zadane formulom

$$
f(x)=A \sin (g(x)),
$$

gdje je $A$ realna konstanta i $g: \mathbb{R} \rightarrow\langle 0,+\infty\rangle$ funkcija oblika (3) ili (4), sastoji se u tome da se prvo skicira graf funkcije $g$ te da se pomoću njega grafički odrede točke presjeka funkcije $f$ i koordinatnih osi te lokalni ekstremi. Postupak za skiciranje grafa funkcije $f$ na segmentu $[a, b]$ sastoji se od sljedećih koraka:

1. Nacrtati graf funkcije $g$ na segmentu $[a, b]$. 
2. Na grafu $g$ odrediti točke $A_{i}, i \in \mathbb{N}$ čije ordinate su oblika

$$
g\left(x_{i}\right)=\frac{\pi}{2} \cdot k, \quad k \in \mathbb{Z},
$$

a apscise $x_{i} \in[a, b]$.

3. Spustiti okomice iz točaka $A_{i}$ na pravce $y=A, y=-A$ i $y=0$. Točke njihova presjeka su lokalni ekstremi ili nultočke funkcije $f$ na segmentu $[a, b]$. Za tako dobivene točke možemo dati sljedeće zaključke:

(a) U točkama

$$
x_{i}=g^{-1}\left(\frac{\pi}{2}+2 k \pi\right)=g^{-1}\left(\frac{\pi}{2}(1+4 k)\right), \quad k \in \mathbb{Z},
$$

funkcija $f$ postiže maksimalnu vrijednost $A$. Točke $x_{i}$ dobivene su kao presjek pravca $y=A$ i okomica spuštenih iz točaka $A_{i}\left(x_{i}, \frac{\pi}{2}+2 k \pi\right), k \in \mathbb{Z}$.

(b) U točkama

$$
x_{i}=g^{-1}\left(\frac{3 \pi}{2}+2 k \pi\right)=g^{-1}\left(\frac{\pi}{2}(3+4 k)\right), \quad k \in \mathbb{Z},
$$

funkcija $f$ postiže minimalnu vrijednost $-A$. Točke $x_{i}$ dobivene su kao presjek pravca $y=-A$ i okomica spuštenih iz točaka $A_{i}\left(x_{i}, \frac{3 \pi}{2}+2 k \pi\right), k \in \mathbb{Z}$.

(c) U točkama

$$
x_{i}=g^{-1}(k \pi), \quad k \in \mathbb{Z},
$$

funkcija $f$ siječe os apscisa. Točke $x_{i}$ dobivene su kao presjek pravca $y=0$ i okomica spuštenih iz točaka $A_{i}\left(x_{i}, k \pi\right), k \in \mathbb{Z}$.

4. Odrediti točku presjeka funkcije $f$ i osi $y$.

5. Skicirati krivulju koja prolazi točkama dobivenim u trećem i četvrtom koraku. To je graf funkcije $f$.

Napomena: Gore opisanim postupkom, na segmentu $[a, b]$ koji bi trebao sadržavati barem dvije točke definirane $u$ trećem koraku te točku 0 definiranu u četvrtom koraku, može se dobiti i graf funkcije oblika $f(x)=A \sin (g(x))$ i $f(x)=A \cos (g(x))$, gdje je $A$ realna konstanta. Stoga, za segment $[a, b]$ nužno je da postoji cijeli broj $k$ takav da vrijedi $g([a, b]) \supseteq\left[\frac{k \pi}{2}, \frac{(k+1) \pi}{2}\right]$. 
Zadatak 1. Skicirati graf funkcije $f(x)=\sin \left(1+\frac{x^{2}}{2}\right)$ na segmentu $[-5,5]$.

\section{Rješenje}

1. Nacrtati graf funkcije $g(x)=1+\frac{x^{2}}{2}$ na zadanom segmentu.

2. Na grafu $g$ odrediti točke čije su ordinate: $\frac{\pi}{2}, \pi, \frac{3 \pi}{2}, 2 \pi, \frac{5 \pi}{2}, 3 \pi, \frac{7 \pi}{2}$.

3. Iz točaka, određenih u drugom koraku, čije su ordinate $\frac{\pi}{2}, \frac{5 \pi}{2}$, spustiti okomice na pravac $y=1$; čije su ordinate $\frac{3 \pi}{2}, \frac{7 \pi}{2}$, spustiti okomice na pravac $y=-1$ te onih čije su ordinate $\pi, 2 \pi, 3 \pi$ spustiti okomice na pravac $y=0$.

4. Točka presjeka funkcije $f$ i osi $y$ je $(0,0.8)$.

5. Kroz točke dobivene u trećem i četvrtom koraku skicirati krivulju. Ta krivulja je graf funkcije $f$.

Na slici 1 ilustriran je postupak skiciranja grafa funkcije $f(x)=$ $\sin \left(1+\frac{x^{2}}{2}\right)$ na segmentu $[-5,5]$.

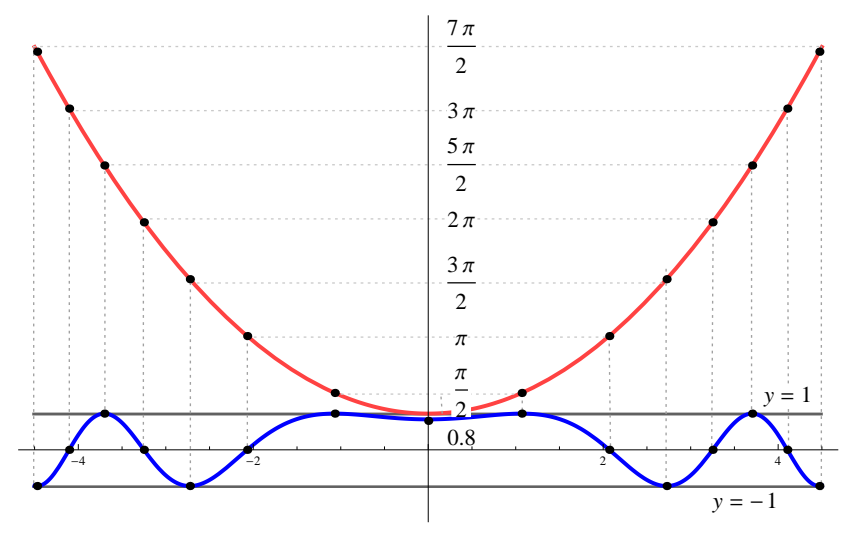

Slika 1: Graf funkcije $f(x)=\sin \left(1+\frac{x^{2}}{2}\right)$ na segmentu $[-5,5]$. Pomoćni graf funkcije $x \mapsto 1+\frac{x^{2}}{2}$ prikazan je crvenom, pravci $y=1$ i $y=-1$ sivom, a graf funkcije $f$ plavom bojom.

Primjenom istog postupka riješite sljedeće zadatke:

Zadatak 2. Skicirati graf funkcije $f(x)=\cos \left(1+x^{2}\right)$ na segmentu $[-2.8,2.8]$.

Zadatak 3. Skicirati graf funkcije $f(x)=\sin \left(e^{\frac{x}{5}}\right)$ na segmentu $[0,13]$.

Rješenje drugog i trećeg zadatka prikazano je na slici 2 odnosno slici 3 


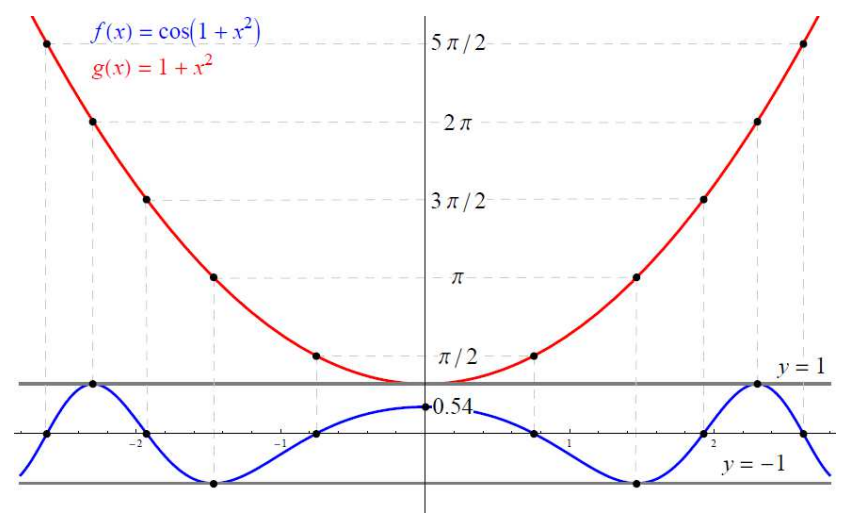

Slika 2: Graf funkcije $f(x)=\cos \left(1+x^{2}\right)$ na segmentu $[-2.8,2.8]$. Pomoćni graf funkcije $x \mapsto 1+x^{2}$ prikazan je crvenom, pravci $y=1$ i $y=-1$ sivom, a graf funkcije $f$ plavom bojom.

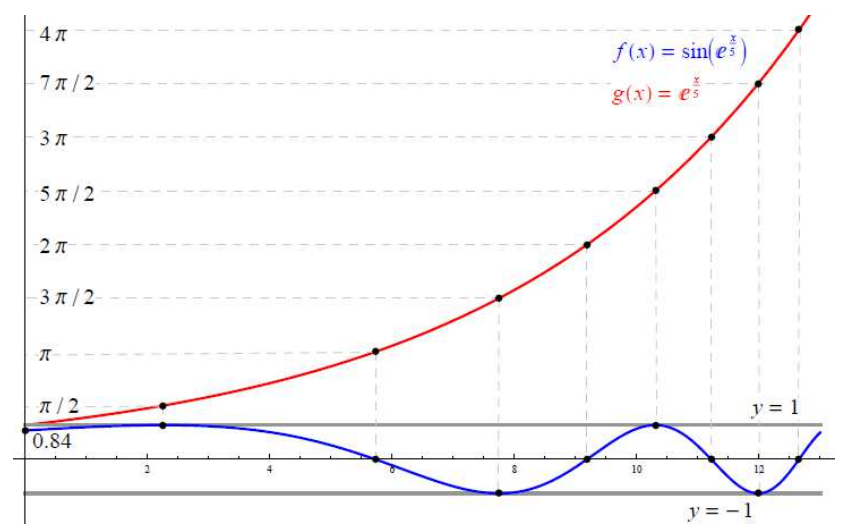

Slika 3: Graf funkcije $f(x)=\sin \left(e^{\frac{x}{5}}\right)$ na segmentu [0,13]. Pomoćni graf funkcije $x \mapsto e^{\frac{x}{5}}$ prikazan je crvenom, pravci $y=1$ i $y=-1$ sivom, a graf funkcije $f$ plavom bojom.

\section{$3 \quad$ Graf funkcije oblika $f(x)=g(x) \cdot \sin (\omega x+\varphi)$}

Neka je $g: \mathbb{R} \rightarrow\langle 0,+\infty\rangle$ funkcija oblika (3) ili (4). Pretpostavimo da na nekom segmentu $[a, b] \subset \mathbb{R}$ želimo skicirati graf funkcije $f: \mathbb{R} \rightarrow \mathbb{R}$ zadane formulom

$$
f(x)=g(x) \cdot \sin (\omega x+\varphi)
$$


gdje su $\omega>0$ i $\varphi \in[-\pi, \pi]$ realne konstante.

Budući da vrijedi $g(x)>0($ tj. $g(x)=|g(x)|)$ i $|\sin (\omega x+\varphi)| \leq 1$ za sve $x \in \mathbb{R}$, vrijedi $g(x)|\sin (\omega x+\varphi)| \leq g(x)$, a odavde je

$$
|g(x)| \cdot|\sin (\omega x+\varphi)|=|f(x)| \leq g(x),
$$

to jest

$$
-g(x) \leq f(x) \leq g(x), \quad x \in \mathbb{R},
$$

što znači da se graf funkcije $f$ nalazi između grafova funkcija $g \mathrm{i}-g$.

Nultočke funkcije $f$ nije teško naći. Budući da funkcija $g$ ima samo pozitivne vrijednosti, vrijedi $f(x)=0 \Longleftrightarrow \sin (\omega x+\varphi)=0$. Dakle, skup $N_{f}$ nultočaka funkcije $f$ podudara se sa skupom nultočaka $N_{h}$ harmonijske funkcije $h(x)=\sin (\omega x+\varphi)$, pa prema [4, str. 23] vrijedi

$$
N_{f}=N_{h}=\left\{\frac{k \pi-\varphi}{\omega}: k \in \mathbb{Z}\right\}
$$

Funkcija $h$, čiji je temeljni period $T=\frac{2 \pi}{\omega}$, postiže maksimalnu vrijednost 1 u točkama

$$
x_{k}^{+}=-\frac{\varphi}{\omega}+\frac{T}{4}+\frac{2 k \pi}{\omega}=\frac{\pi-2 \varphi}{2 \omega}+\frac{2 k \pi}{\omega}, \quad k \in \mathbb{Z},
$$

a minimalnu vrijednost $-1 \mathrm{u}$ točkama

$$
x_{k}^{-}=-\frac{\varphi}{\omega}+\frac{3 T}{4}+\frac{2 k \pi}{\omega}=\frac{3 \pi-2 \varphi}{2 \omega}+\frac{2 k \pi}{\omega}, \quad k \in \mathbb{Z}
$$

(vidi [4, str. 24]). Ako je $x$ točka maksimuma funkcije $h$, tada vrijedi $f(x)=g(x)$, a ako je $x$ točka minimuma funkcije $h$, tada vrijedi $f(x)=$ $-g(x)$. Drugim riječima, graf funkcije $f$ siječe graf funkcije $g$ isključivo $\mathrm{u}$ točkama $x_{k}^{+}$odnosno graf funkcije $-g$ isključivo u točkama $x_{k}^{-}$za $k \in \mathbb{Z}$. Odredimo derivaciju funkcije $f$ (koju dobivamo prema pravilima za određivanje derivacije umnoška i derivacije složene funkcije, vidi [4, str. 32]):

$$
f^{\prime}(x)=g^{\prime}(x) \sin (\omega x+\varphi)+\omega g(x) \cos (\omega x+\varphi) .
$$

Kako je $h\left(x_{k}^{+}\right)=\sin \left(\omega x_{k}^{+}+\varphi\right)=1$ i vrijedi $\sin ^{2}\left(\omega x_{k}^{+}+\varphi\right)+\cos ^{2}\left(\omega x_{k}^{+}+\right.$ $\varphi)=1$, dobivamo $\cos \left(\omega x_{k}^{+}+\varphi\right)=0$, pa vrijedi

$$
f^{\prime}\left(x_{k}^{+}\right)=g^{\prime}\left(x_{k}^{+}\right), \quad k \in \mathbb{Z} .
$$

Potpuno analogno dobivamo

$$
f^{\prime}\left(x_{k}^{-}\right)=g^{\prime}\left(x_{k}^{-}\right), \quad k \in \mathbb{Z} .
$$


Odavde slijedi da se grafovi funkcija $f$ i $g$ odnosno $-g$ dodiruju u točkama $x_{k}^{+}$odnosno $x_{k}^{-}$za $k \in \mathbb{Z}, \mathrm{tj}$. u tim točkama se sijeku i imaju zajedničku tangentu.

Napomenimo da se lokalni ekstremi funkcije $f$ općenito ne podudaraju s lokalnim ekstremima harmonijske funkcije $h$. Naime, u tim je točkama vrijednost funkcije $f^{\prime}$ jednaka vrijednosti funkcije $g^{\prime}$ koja je, za funkciju $g$ oblika (4), uvijek različita od nule (eksponencijalna funkcija je strogo rastuća), pa točke $x_{k}^{+}$i $x_{k}^{-}$općenito ne ispunjavaju nužan uvjet za lokalni ekstrem, a to je da derivacija $f^{\prime} \mathrm{u}$ tim točkama ima vrijednost nula (vidi 4, str. 33]). Za razliku od nultočaka, lokalne ekstreme funkcije $f$ nije lako odrediti. Također, nizovi lokalnih minimuma odnosno lokalnih maksimuma funkcije $f$ općenito nisu aritmetički, kao što su to kod funkcije $h$.

Na osnovu dobivenih rezultata možemo dati sljedeći postupak za skiciranje funkcije $f$ na segmentu $[a, b]$, koji bi trebao sadržavati najmanje po jednu točku $x_{k}^{+}$i $x_{k}^{-}$.

1. Skicirati (pomoćne) grafove funkcija $g \mathrm{i}-g$ na segmentu $[a, b]$.

2. Ucrtati točke iz skupa $N_{f} \cap[a, b]$ na os apscisa.

3. Ucrtati točke grafa funkcije $g \mathrm{~s}$ apscisom $x_{k}^{+} \in[a, b], k \in \mathbb{Z}$ te povući tangente u tim točkama.

4. Ucrtati točke grafa funkcije $-g$ s apscisom $x_{k}^{-} \in[a, b], k \in \mathbb{Z}$ te povući tangente u tim točkama.

5. Skicirati krivulju koja prolazi točkama dobivenim u drugom koraku, dodiruje graf funkcije $g$ u točkama dobivenim u trećem koraku te graf funkcije $-g$ u točkama dobivenim u četvrtom koraku. To je graf funkcije $f$.

Napomena: Gore opisanim postupkom može se dobiti i graf funkcije oblika $f(x)=g(x) \sin (\omega x+\varphi)$ i $f(x)=g(x) \cos (\omega x+\varphi)$ gdje su $\omega \neq 0$ i $\varphi$ proizvoljni realni brojevi. Naime, svaka se funkcija oblika $\sin (\omega x+\varphi)$ ili $\cos (\omega x+\varphi)$ može zapisati kao harmonijska funkcija (vidi [4, str. 23]).

Zadatak 4. Skicirati graf funkcije $f(x)=\left(1+\frac{x^{2}}{10}\right) \cdot \sin x$ na segmentu $[0,3 \pi]$.

\section{Rješenje}

1. Nacrtati grafove funkcija $g$ i $-g$ tj. $x \mapsto 1+\frac{x^{2}}{10}$ i $x \mapsto-1-\frac{x^{2}}{10}$ na zadanom segmentu.

2. Ucrtati nultočke funkcije $f: 0, \pi, 2 \pi, 3 \pi$. 
3. Ucrtati točke s apscisom $\frac{\pi}{2}, \frac{5 \pi}{2}$ na grafu funkcije $g$ te kroz njih povući tangente.

4. Ucrtati točku s apscisom $\frac{3 \pi}{2}$ na grafu funkcije $-g$ te kroz nju povući tangentu.

5. Na ranije opisan način iz podataka dobivenih u prethodnim koracima skicirati krivulju. Ta krivulja je graf funkcije $f$.

Na slici 4 ilustriran je postupak skiciranja grafa funkcije $f(x)=\left(1+\frac{x^{2}}{10}\right)$. $\sin x$ na segmentu $[0,3 \pi]$.

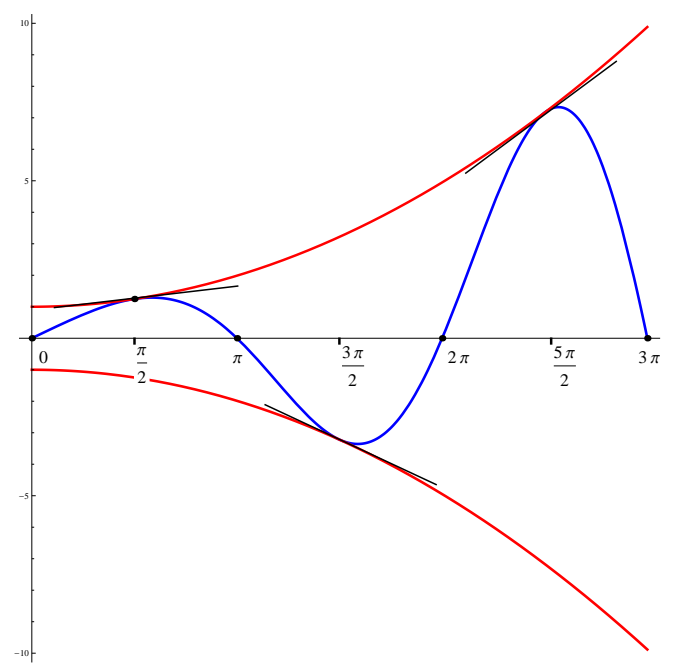

Slika 4: Graf funkcije $f(x)=\left(1+\frac{x^{2}}{10}\right) \cdot \sin x$ na segmentu [0,3 $\pi$. Pomoćni grafovi funkcija $x \mapsto 1+\frac{x^{2}}{10}$ i $x \mapsto-1-\frac{x^{2}}{10}$ prikazani su crvenom, a graf funkcije $f$ plavom bojom.

Primjenom istog postupka riješite sljedeće zadatke:

Zadatak 5. Skicirati graf funkcije $f(x)=\left(1+x^{2}\right) \cdot \cos (2 x)$ na segmentu $[-7,7]$.

Zadatak 6. Skicirati graf funkcije $f(x)=e^{\frac{x}{5}} \cdot \sin (x+1)$ na segmentu $[-8,8]$.

Rješenje petog i šestog zadatka prikazano je na slici 5 odnosno slici 6 . 


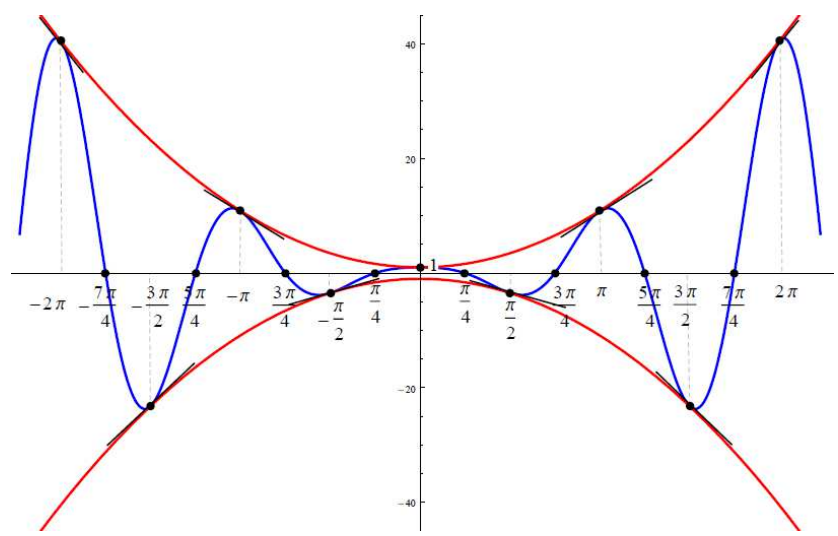

Slika 5: Graf funkcije $f(x)=\left(1+x^{2}\right) \cdot \cos (2 x)$ na segmentu $[-7,7]$. Pomoćni grafovi funkcija $x \mapsto 1+x^{2}$ i $x \mapsto-1-x^{2}$ prikazani su crvenom, a graf funkcije $f$ plavom bojom.

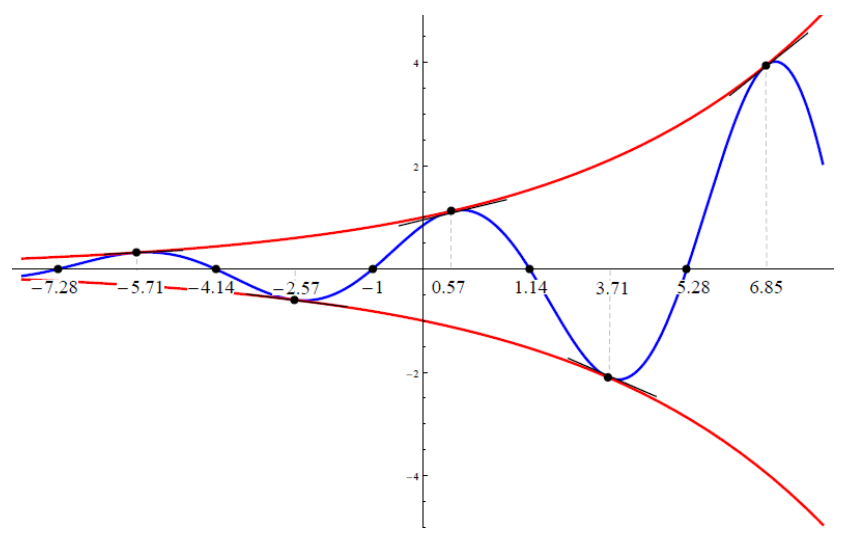

Slika 6: Graf funkcije $f(x)=e^{\frac{x}{5}} \cdot \sin (x+1)$ na segmentu $[-8,8]$. Pomoćni grafovi funkcija $x \mapsto e^{\frac{x}{5}}$ i $x \mapsto-e^{\frac{x}{5}}$ prikazani su crvenom, a graf funkcije $f$ plavom bojom.

\section{Literatura}

[1] B. Dakić, N. Elezović, Matematika 2, 1. dio: udžbenik i zbirka zadataka za 2. razred prirodoslovno-matematičke gimnazije, Element, 2014 .

[2] B. Dakić, N. Elezović, Matematika 2, 2. dio: udžbenik i zbirka za- 
dataka za 2. razred prirodoslovno-matematičke gimnazije, Element, 2014.

[3] B. Dakić, N. Elezović, Matematika 3, udžbenik i zbirka zadataka za 3. razred prirodoslovno-matematičke gimnazije, Element, 2014.

[4] B. Kovačić, L. Marohnić, T. Strmečki, Repetitorij matematike za studente elektrotehnike, Tehničko veleučilište u Zagrebu, Zagreb, 2016.

Mandi Orlić Bachler

Tehničko veleučilište u Zagrebu, Graditeljski odjel, Av. V. Holjevca 15, Zagreb

E-mail adresa: mandi.orlic@tvz.hr

Luka Marohnić

Tehničko veleučilište u Zagrebu, Elektrotehnički odjel, Konavoska 2, Zagreb

E-mail adresa: luka.marohnic@tvz.hr

Martina Dokić

studentica preddiplomskog stručnog studija graditeljstva, Tehničko veleučilište u Zagrebu, Graditeljski odjel, Av. V. Holjevca 15, Zagreb

E-mail adresa: martina.dokic@tvz.hr 